\title{
Radiation-Induced Correlation between Molecules Nearby Metallic Antenna Array
}

\author{
Yoshiki Osaka, Nobuhiko Yokoshi, and Hajime Ishihara \\ Department of Physics and Electronics, Osaka Prefecture University, 1-1 Gakuen-cho, Sakai, Osaka 599-8531, Japan \\ Correspondence should be addressed to Nobuhiko Yokoshi; yokoshi@pe.osakafu-u.ac.jp
}

Received 1 June 2015; Accepted 15 September 2015

Academic Editor: Shah N. Burokur

Copyright ( 2015 Yoshiki Osaka et al. This is an open access article distributed under the Creative Commons Attribution License, which permits unrestricted use, distribution, and reproduction in any medium, provided the original work is properly cited.

\begin{abstract}
We theoretically investigate optical absorption of molecules embedded nearby metallic antennas by using discrete dipole approximation method. It is found that the spectral peak of the absorption is shifted due to the radiation-induced correlation between the molecules. The most distinguishing feature of our work is to show that the shift is largely enhanced even when the individual molecules couple with localized surface plasmons near the different antennas. Specifically, we first consider the case that two sets of dimeric gold blocks with a spacing of a few nanometers are arranged and reveal that the intensity and spectral peak of the optical absorption strongly depend on the position of the molecules. In addition, when the dimeric blocks and the molecules are periodically arranged, the peak shift is found to increase up to $\sim 1.2 \mathrm{meV}(300 \mathrm{GHz})$. Because the radiation-induced correlation is essential for collective photon emission, our result implies the possibility of plasmon-assisted superfluorescence in designed antenna-molecule complex systems.
\end{abstract}

\section{Introduction}

Optically illuminated metallic nanostructure, such as nanoparticles and nanoblocks, generates an extremely localized electric field due to localized surface plasmon resonance (LSPR), by which we can make individual molecules effectively couple with weak light [1]. In addition, the intensity gradient of the localized field becomes steep enough to break long-wavelength approximation and excite dipole-forbidden transitions even in a single molecule [2-4]. These features remind us of sophisticated antenna systems for photons and have attracted increased attention from various scientific fields with a view to efficient reaction fields [5-7]. We have theoretically examined optical responses of antennamolecule coupled systems and found that, for certain set of parameters, one can efficiently excite only the molecule with the antenna excitation inhibited (energy transparency effect) $[8,9]$. Actually, similar effect is experimentally verified using gold nanoantenna system [10]. Such a transparency is explained by considering the quantum interference between the molecular polarization and the plasmon, and it can be applied also to the nonlinear response by a few photons [11].
Besides, it is theoretically proposed that the plasmonassisted enhancement of light-molecule interaction can be applied to cooperative photoemission such as superradiance and superfluorescence $[12,13]$. According to Dicke's theory, the ensemble of emitters can radiate coherent light after creating a macroscopic dipole moment through the incident and radiation fields $[14,15]$. Then, in general, cooperative photoemission requires high-density emitters. Actually, in $[12,13]$, all the molecules are assumed to lie under the common localized field. However, if the molecules are efficiently excited by using the energy transparency effect, the radiated field from the molecule should be enlarged. Then, such plasmon-assisted radiations can enhance intermolecule correlation.

In the present work, we numerically analyze the optical absorption of molecules near a metallic nanostructure by using discrete dipole approximation (DDA) method [16-18] and investigate the correlation between the molecules. Here it is assumed that the metallic structure consists of dimeric gold nanoblocks with a spacing of a few nanometers. In such a system, the intensity of the localized electric field becomes 


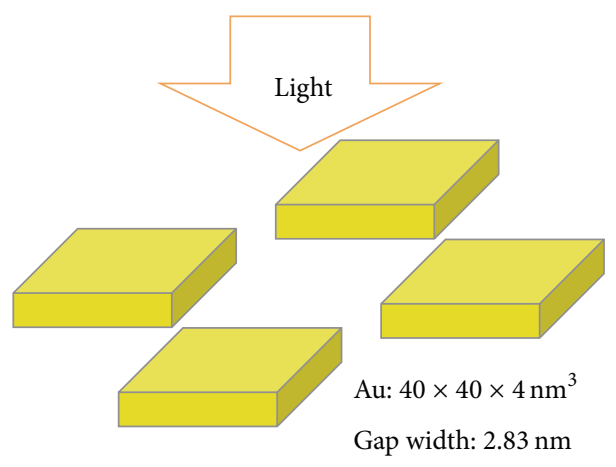

(a)

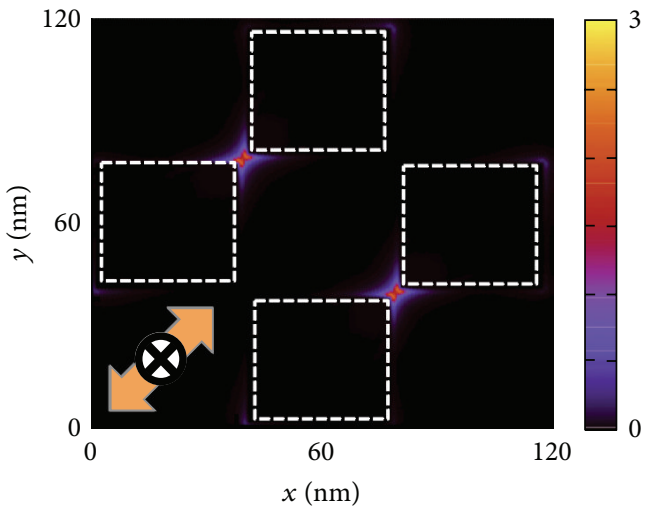

(c)

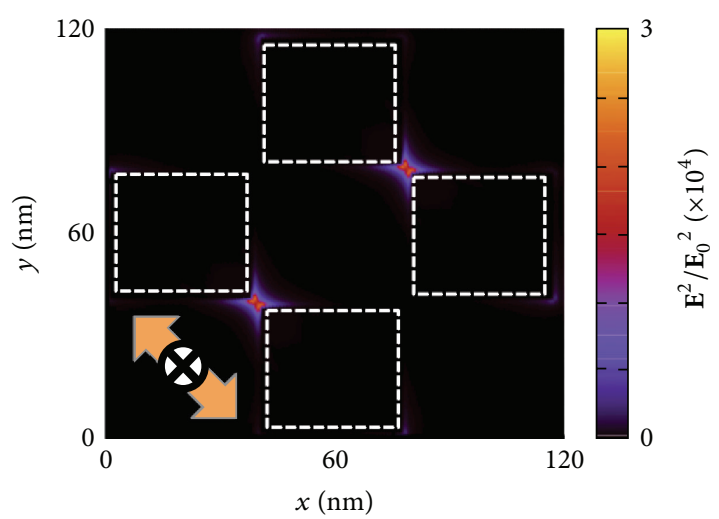

(b)

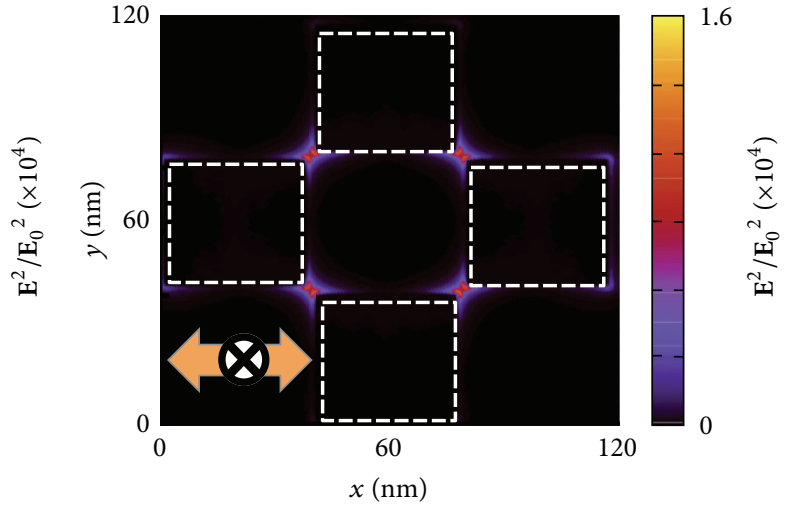

(d)

Figure 1: (a) Schematic figure of the metallic nanoblocks: we arrange four gold nanoblocks with a small spacing $2.83 \mathrm{~nm}$; the size of each nanoblock is $40 \times 40 \times 4 \mathrm{~nm}^{3}$. (b) The profile of the electric field intensity in the absence of the molecules: the two-way orange arrow indicates the direction of the polarization of the incident field; one can see that the total electric field is strongly enhanced at the spacing and produces "hot spots" for the molecules. (c, d) The profile of the total electric fields is plotted when the direction of the polarization is changed; one can see that the position and the intensity are changed with the direction.

$\sim 10^{5}$ times larger than that of the incident light [19]. In addition, the periodic array of the dimeric blocks is also produced experimentally $[20,21]$. It is found that the spectral peak of the optical absorption becomes largely shifted even when the molecules couple with different plasmons. We also find that radiation-induced correlation is further enhanced when the dimeric blocks and molecules are periodically arranged. The quantitative study of the intermolecule correlation is essential to explore the cooperative photoemission in the metallic antenna-molecule coupled system and may contribute to designing future photoemission devices.

\section{Model and Method}

The system under consideration is shown in Figure 1(a). The size of each block is $40 \times 40 \times 4 \mathrm{~nm}^{3}$ and the spacing between the blocks is $2.83 \mathrm{~nm}$. For a clear demonstration, we have considered the blocks to be sufficiently thin so that the mode volume of the localized light fields becomes comparable to the molecular volume. In order to obtain the optical absorption, we solve the discretized integral form of Maxwell's equation within DDA [16-18]. In this calculation, we divide the whole space containing the metal blocks into small cubic cells, where microscopic quantities such as the electric field and polarization in each cell are averaged. The integral equation is given by

$$
\mathbf{E}_{i}=\mathbf{E}_{i}^{0}+\sum_{j=1}^{n} \mathbf{G}_{i, j}^{f} \mathbf{P}_{j} V_{j}+\left(\mathbf{M}_{i}-\mathbf{L}_{i}\right) \mathbf{P}_{i},
$$

where $n$ represents the total number of cells and $i, j$ are the cell number. The incident field $\mathbf{E}_{i}^{0}$ is linearly polarized along the direction of the orange two-way arrows in Figures 1(b)$1(d)$, and the polarization in each cell is connected with the total electric field $\mathbf{E}_{i}$ as

$$
\mathbf{P}_{j}=\chi_{j} \mathbf{E}_{j}
$$

Here, $\chi_{j}$ and $V_{j}$ are the optical susceptibility and the volume of the $j$ th cell, respectively. The free space Green's function $\mathbf{G}_{i, j}^{f}$ has both transverse and longitudinal electromagnetic 


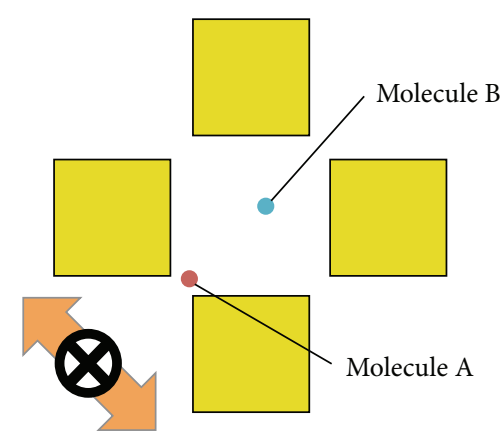

(a)

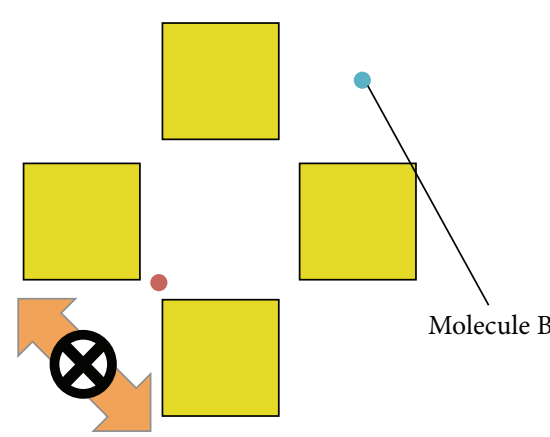

(b)

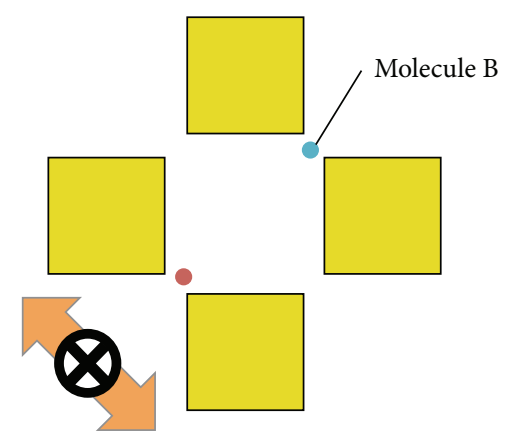

(c)
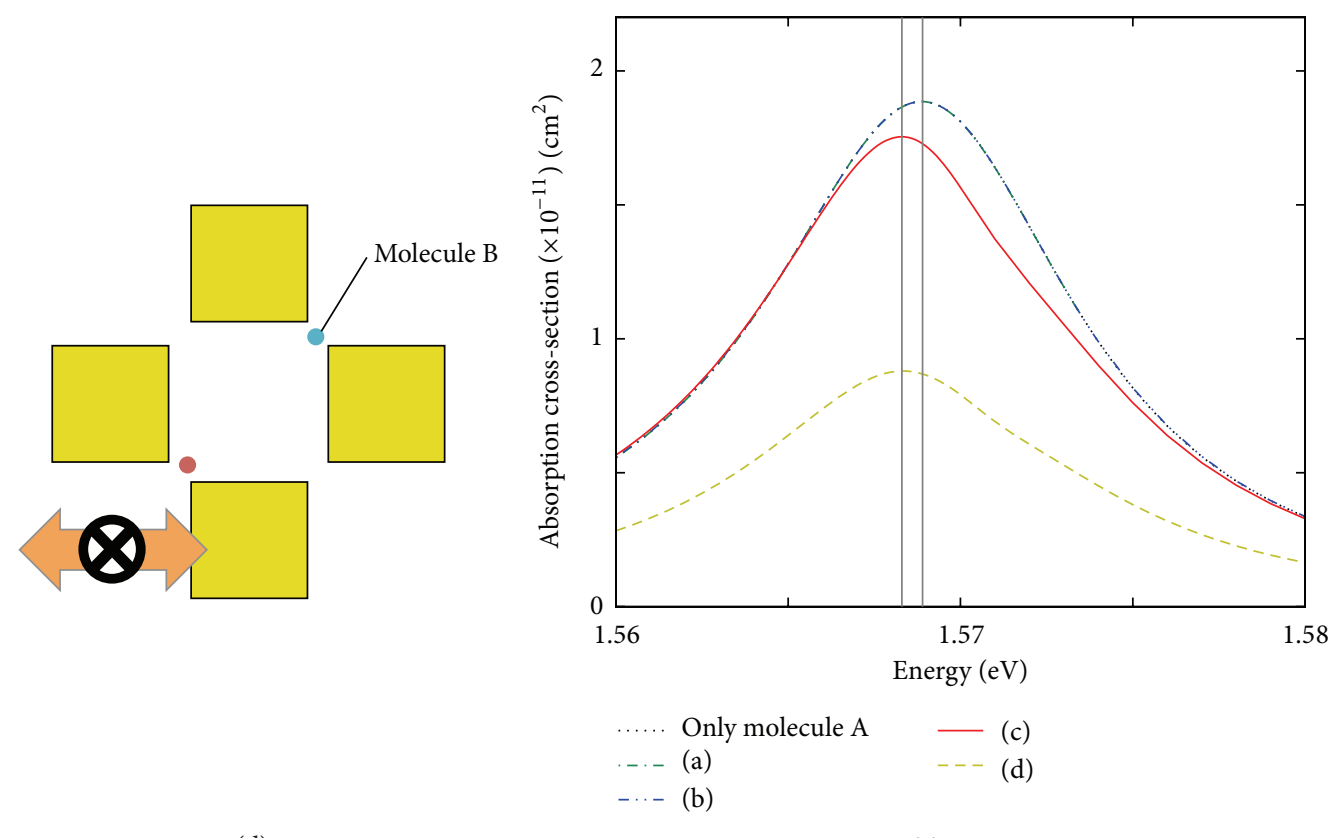

(d)

(e)

FIgURE 2: $(\mathrm{a}-\mathrm{d})$ Top view of the position of two molecules and metal blocks: the polarization direction of the incident field is represented by two-way orange arrow. $(\mathrm{a}-\mathrm{c})$ One of the molecules is fixed (red circle), while the other one (blue circle) is placed in different positions. (d) We alter the polarization direction in the same configuration as (c). (e) Absorption cross-section of the molecule (red circle) is plotted against the incident field energy for different arrangement of molecules. The black dotted line represents the absorption for the case where only one molecule (red circle) exists. One can see the large peak shift $(\sim 0.6 \mathrm{meV})$ due to the intermolecule interaction, only when both of the molecules are located in the hot spots (c, d).

components. The term including $\mathbf{M}_{i}-\mathbf{L}_{i}$ denotes the selfinteraction, which is introduced in order to avoid unphysical divergence and can be calculated analytically as $[22,23]$

$$
\begin{aligned}
\mathbf{M}_{i} & =\frac{8 \pi}{3} \mathbf{I}[(1-i k a) \exp (i k a)-1] \\
\mathbf{L}_{i} & =\frac{4 \pi}{3} \mathbf{I} .
\end{aligned}
$$

Here the parameter $a$ characterizes the size of the unit cell and $k=\omega / c$ is the wave number with $\omega$ being the frequency of the incident field.
The unit cell in the metal blocks has a Drude-type dielectric function

$$
\chi_{i}^{\mathrm{m}}=\varepsilon_{\mathrm{bg}}^{\mathrm{m}}-\varepsilon_{\mathrm{bg}}-\frac{\left(\hbar \omega_{p}\right)^{2}}{\left[(\hbar \omega)^{2}+i \hbar \omega\left(\hbar \gamma_{\mathrm{b}}+\hbar \nu_{F} / D_{\mathrm{eff}}\right)\right]},
$$

where the background dielectric constant of the gold is set to be $\varepsilon_{\mathrm{bg}}^{\mathrm{m}}=12.0$ and the environmental background dielectric constant is set to be $\varepsilon_{\mathrm{bg}}=1.0$, that is, the dielectric constant of vacuum. As for the other parameters in the gold blocks, we employ the ones in $[24,25]$ : the plasma frequency $\hbar \omega_{p}=$ $8.958 \mathrm{eV}$, the nonradiative damping constant $\hbar \gamma_{\mathrm{b}}=72.3 \mathrm{meV}$, the mean free path of electron $D_{\text {eff }}=20 \mathrm{~nm}$, and the Fermi 


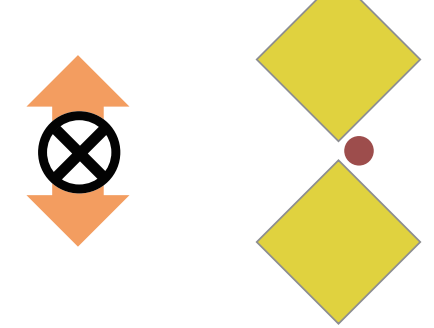

(a) One-dimer

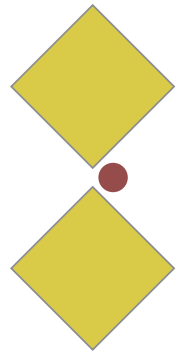

(b) Two-dimer (one molecule)

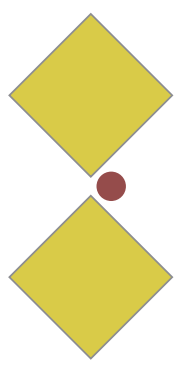

(c) Two-dimer (two molecules)
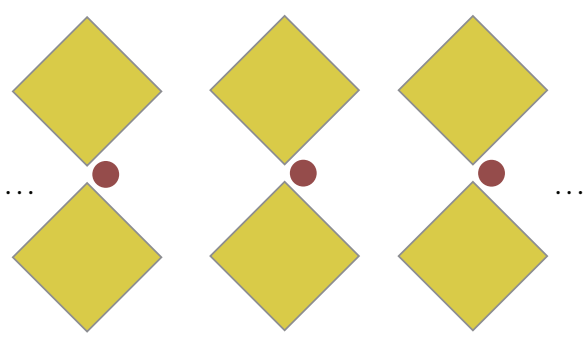

(d) $1 \mathrm{D}$ periodic structure

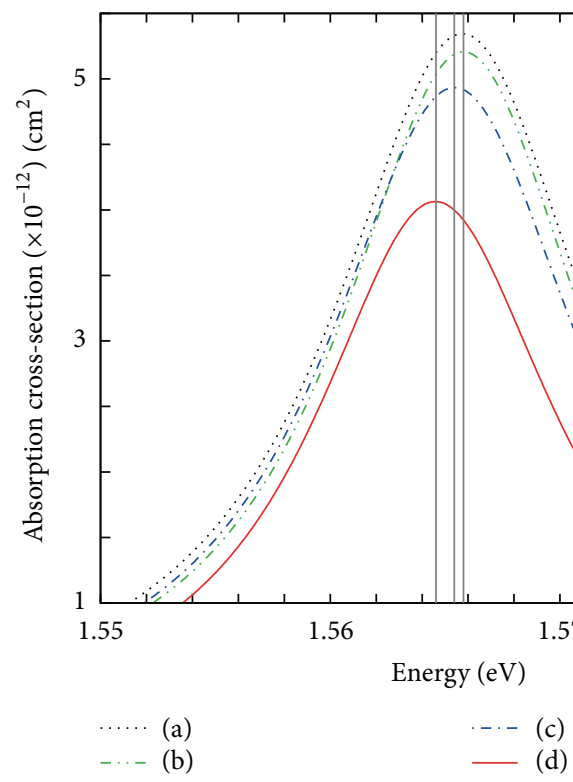

(e)

Figure 3: (a) Top view of the arrangement of the dimeric antennas and the molecule; here the spacing between the blocks is changed to be $3 \mathrm{~nm}$ according to the convenience in the calculation. (b-c) Top view: two sets of the dimeric antennas and one and two molecules, respectively. (d) Top view of the periodic $1 \mathrm{D}$ array of the dimeric antenna-molecule coupled system. (e) The absorption cross-section is plotted for each arrangement pattern; one can see that, in the $1 \mathrm{D}$ periodic array, the largest red-shift $\sim 1.2 \mathrm{meV}$ is induced due to the radiation-induced intermolecule correlation, which exceeds the one due to the antenna-molecule coupling.

velocity $v_{F}=1.4 \times 10^{6} \mathrm{~m} / \mathrm{s}$. As for the susceptibility of the molecule, we assume it to be Lorentzian:

$$
\chi_{j}^{\mathrm{m}}=\frac{\left(|d|^{2} / V_{j}\right)}{\left(\hbar \Omega^{\mathrm{m}}-\hbar \omega-i \gamma\right)},
$$

where $d=9.7$ Debye is the dipole moment, $\hbar \Omega=1.57 \mathrm{eV}$ is the resonant energy, and $\hbar \gamma=1 \mathrm{meV}$ is the nonradiative damping constant. We have employed these parameters so that the molecular system becomes resonant to LSPR of the gold blocks, which is necessary in order to introduce the energy transparency effect $[8,9]$.

In this work, we mainly calculate the absorption crosssection of molecule according to the definition

$$
\sigma_{\mathrm{abs}}=4 \pi \frac{\omega}{c} \int_{V} d^{3} \mathbf{r} \frac{|\mathbf{E}(\mathbf{r})|^{2}}{\left|\mathbf{E}_{0}(\mathbf{r})\right|^{2}} \operatorname{Im}\{\chi(\mathbf{r})\}
$$


It should be noted that the total electric field is derived by numerically solving the simultaneous equations in (1) and (2); that is, the electric fields in the metallic antenna and the molecule are self-consistently calculated. We will estimate the correlation between molecules from the spectra of the absorption cross-section.

\section{Results}

Before calculating the optical absorption of the molecules, we show the profile of the electric field intensity for the case where only the four gold blocks are set (see Figures 1(b)-1(d)). One can see that electric field is enormously enhanced nearby the gap structure of the dimeric antenna, so-called "hot spot." Therefore, when the molecules are set at the hot spot, the plasmon fields excite the molecules efficiently. Note that the electric field becomes considerably small far from the hot spots and then we can excite plasmons with small correlations in the distant spots.

Subsequently, we investigate the correlation between the two molecules. We arrange the molecules at the position of the red and blue circles in Figures 2(a)-2(d). In Figure 2(e), the calculated absorption cross-sections of molecule A (red circle) are plotted against the energy of the incident light. One can see that when only one molecule is set in the hot spot, the peak of the absorption is red-shifted by $1.1 \mathrm{meV}$ from the resonant energy $\hbar \Omega=1.57 \mathrm{eV}$ (see black dotted line). This shift is caused by the dipole-dipole interaction between the dimeric antenna and the molecule. When another molecule (B) is arranged apart from the hot spot, we cannot see the visible difference in the spectrum (green dashed-dotted line and blue dashed-two-dotted line). On the other hand, in case that both of the molecules are set in different hot spots, the absorption cross-section is further red-shifted (red continuous line). We consider that this additional shift originates from the molecule-molecule coupling via the radiation field, because the interference between the plasmons in the distant hot spots has been already included in the case with single molecule. This is the essential difference from the previous work $[12,13]$. The additional shift reaches $0.6 \mathrm{meV}(150 \mathrm{GHz})$ that is comparable to the one by the antenna-molecule coupling. The large intermolecule correlation appears only when both of them are excited with high efficiency due to energy transparency effect, and then the plasmon-molecule interference plays the essential role also in the intermolecule correlation. Besides, when the polarization direction of the incident field is altered, the number of hot spots is also altered. Seeing the yellow dashed line in Figure 2(e), the comparable shift is observed even though the absorption cross-section decreases due to the decrease of the effective electric field in one hot spot.

The intermolecule correlation can be further enhanced by periodically arranging the dimeric antenna-molecule complex. In order to calculate the optical absorption in such a system, we apply the periodic boundary condition to DDA calculation following [26]. In order to apply the boundary condition, we have changed slightly the spacing between the nanoblocks into $3 \mathrm{~nm}$. Figures 3(a)-3(d) show the arrangement of the metallic antenna and molecules. In
Figure 3(e), we plot the absorption spectra of one molecule for different arrangements. One can see that the peak shift of the absorption spectrum is no more than $0.4 \mathrm{meV}$ when we add the second set of dimeric antenna and molecule. On the other hand, in case of 1D periodic array structure, the peak shit is increased up to $\sim 1.2 \mathrm{meV}$ ( $300 \mathrm{GHz}$ ), which exceeds the one due to dipole-dipole interaction between one dimeric antenna and one molecule.

\section{Conclusion}

With the use of discrete dipole approximation method, we have numerically investigated optical absorption of molecules nearby metallic antennas. We find that large radiation-induced correlation between the molecules can appear even when they couple with different plasmons. The essence of such a large correlation is energy transparency effect due to quantum interference between the plasmon and molecular polarization. Because of the effect, one of the molecules is effectively excited and radiates a photon that couples to the other molecule. We also have evaluated the intermolecule correlation in periodically arranged antennamolecule complex and found that the correlation can be comparable or greater than the single antenna-molecule correlation.

Although our calculation is based on only linear response theory, the large intermolecule correlation makes us expect introducing collective photoemission. Because the metallic antenna can be designed, it may lead to photoemission devices using controlled superfluorescence. For that aim, we have to calculate optical responses including nonlinear excitations in future work.

\section{Conflict of Interests}

The authors declare that there is no conflict of interests regarding the publication of this paper.

\section{Acknowledgments}

The authors thank Dr. Y. Mizumoto for helpful discussion on numerical calculation by discrete dipole approximation method. This work was partially supported by a Grant-inAid for JSPS Fellows no. 13J09308 and a Grant-in-Aid for Scientific Research (A) no. 24244048 from Japan Society for Promotion of Science (JSPS).

\section{References}

[1] P. Anger, P. Bharadwaj, and L. Novotny, "Enhancement and quenching of single-molecule fluorescence," Physical Review Letters, vol. 96, no. 11, Article ID 113002, 4 pages, 2006.

[2] T. Iida and H. Ishihara, "Unconventional control of excited states of a dimer molecule by a localized light field between metal nanostructures," Physica Status Solidi (A), vol. 206, no. 5, pp. 980-984, 2009.

[3] P. K. Jain, D. Ghosh, R. Baer, E. Rabani, and A. P. Alivisatos, "Near-field manipulation of spectroscopic selection rules on the nanoscale," Proceedings of the National Academy of Sciences of the United States of America, vol. 109, no. 21, pp. 8016-8019, 2012. 
[4] M. Takase, H. Ajiki, Y. Mizumoto et al., "Selection-rule breakdown in plasmon-induced electronic excitation of an isolated single-walled carbon nanotube," Nature Photonics, vol. 7, no. 7, pp. 550-554, 2013.

[5] P. Bharadwaj, B. Deutsch, and L. Novotny, "Optical antennas," Advances in Optics and Photonics, vol. 1, no. 3, pp. 438-483, 2009.

[6] L. Novotny and N. van Hulst, "Antennas for light," Nature Photonics, vol. 5, no. 2, pp. 83-90, 2011.

[7] P. Mühlschlegel, H.-J. Eisler, O. J. F. Martin, B. Hecht, and D. W. Pohl, "Resonant optical antennas," Science, vol. 308, no. 5728, pp. 1607-1609, 2005.

[8] H. Ishihara, A. Nobuhiro, M. Nakatani, and Y. Mizumoto, "Anomalous optical response of metal-molecule coupled system," Journal of Photochemistry and Photobiology A: Chemistry, vol. 221, no. 2-3, pp. 148-153, 2011.

[9] M. Nakatani, A. Nobuhiro, N. Yokoshi, and H. Ishihara, "Model of the photoexcitation processes of a two-level molecule coherently coupled to an optical antenna," Physical Chemistry Chemical Physics, vol. 15, no. 21, pp. 8144-8150, 2013.

[10] Y. Tanaka, H. Ishiguro, H. Fujiwara et al., "Direct imaging of nanogap-mode plasmon-resonant fields," Optics Express, vol. 19, no. 8, pp. 7726-7733, 2011.

[11] Y. Osaka, N. Yokoshi, M. Nakatani, and H. Ishihara, "Enhanced up-conversion of entangled photons and quantum interference under a localized field in nanostructures," Physical Review Letters, vol. 112, no. 13, Article ID 133601, 5 pages, 2014.

[12] V. N. Pustovit and T. V. Shahbazyan, "Cooperative emission of light by an ensemble of dipoles near a metal nanoparticle: the plasmonic Dicke effect," Physical Review Letters, vol. 102, no. 7, Article ID 077401, 4 pages, 2009.

[13] P. A. Huidobro, A. Y. Nikitin, C. González-Ballestero, L. MartínMoreno, and F. J. García-Vidal, "Superradiance mediated by graphene surface plasmons," Physical Review B, vol. 85, no. 15, Article ID 155438, 2012.

[14] R. H. Dicke, "Coherence in spontaneous radiation processes," Physical Review, vol. 93, no. 1, pp. 99-110, 1954.

[15] R. Bonifacio and L. A. Lugiato, "Cooperative radiation processes in two-level systems: superfluorescence," Physical Review $A$, vol. 11, no. 5, article 1507, 1975.

[16] E. M. Purcell and C. R. Pennypacker, "Scattering and absorption of light by nonspherical dielectric grains," The Astrophysical Journal, vol. 186, pp. 705-714, 1973.

[17] M. A. Yurkin and A. G. Hoekstra, "The discrete dipole approximation: an overview and recent developments," Journal of Quantitative Spectroscopy and Radiative Transfer, vol. 106, no. 1-3, pp. 558-589, 2007.

[18] F. Della Sala and S. D'Agostino, Handbook of Molecular Plasmonics, Pan Stanford Publishing, 2013.

[19] E. Hao and G. C. Schatz, "Electromagnetic fields around silver nanoparticles and dimers," The Journal of Chemical Physics, vol. 120, no. 1, pp. 357-366, 2004.

[20] K. Ueno, S. Juodkazis, T. Shibuya et al., "Nanoparticle plasmonassisted two-photon polymerization induced by incoherent excitation source," Journal of the American Chemical Society, vol. 130, no. 22, pp. 6928-6929, 2008.

[21] V. G. Kravets, F. Schedin, and A. N. Grigorenko, "Extremely narrow plasmon resonances based on diffraction coupling of localized plasmons in arrays of metallic nanoparticles," Physical Review Letters, vol. 101, no. 8, Article ID 087403, 2008.
[22] A. D. Yaghjian, "Electric dyadic Green's functions in the source region," Proceedings of the IEEE, vol. 68, no. 2, pp. 248-263, 1980.

[23] J. I. Hage and J. M. Greenberg, "A model for the optical properties of porous grains," Astrophysical Journal, vol. 361, no. 1, pp. 251-259, 1990.

[24] P. B. Johnson and R. W. Christy, "Optical constants of the noble metals," Physical Review B, vol. 6, no. 12, article 4370, 1972.

[25] R. Antoine, P. F. Brevet, H. H. Girault, D. Bethell, and D. J. Schiffrin, "Surface plasmon enhanced non-linear optical response of gold nanoparticles at the air/toluene interface," Chemical Communications, no. 19, pp. 1901-1902, 1997.

[26] B. T. Draine and P. J. Flatau, "Discrete-dipole approximation for periodic targets: theory and tests," Journal of the Optical Society of America A, vol. 25, no. 11, pp. 2693-2703, 2008. 

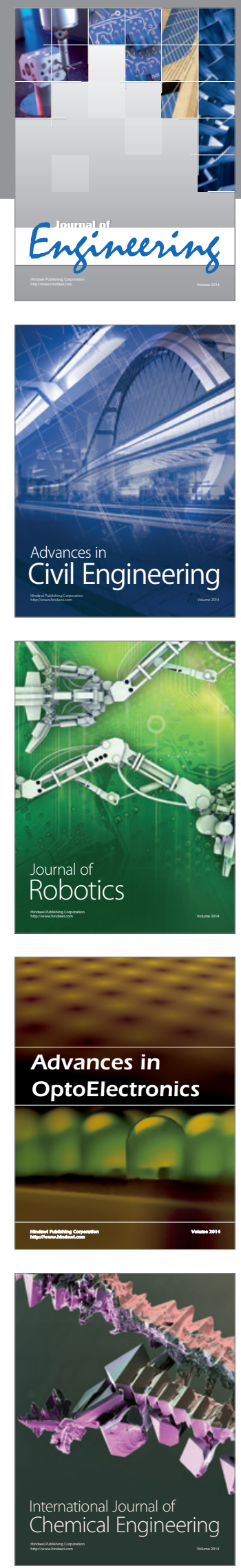

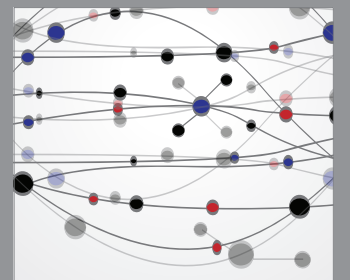

The Scientific World Journal
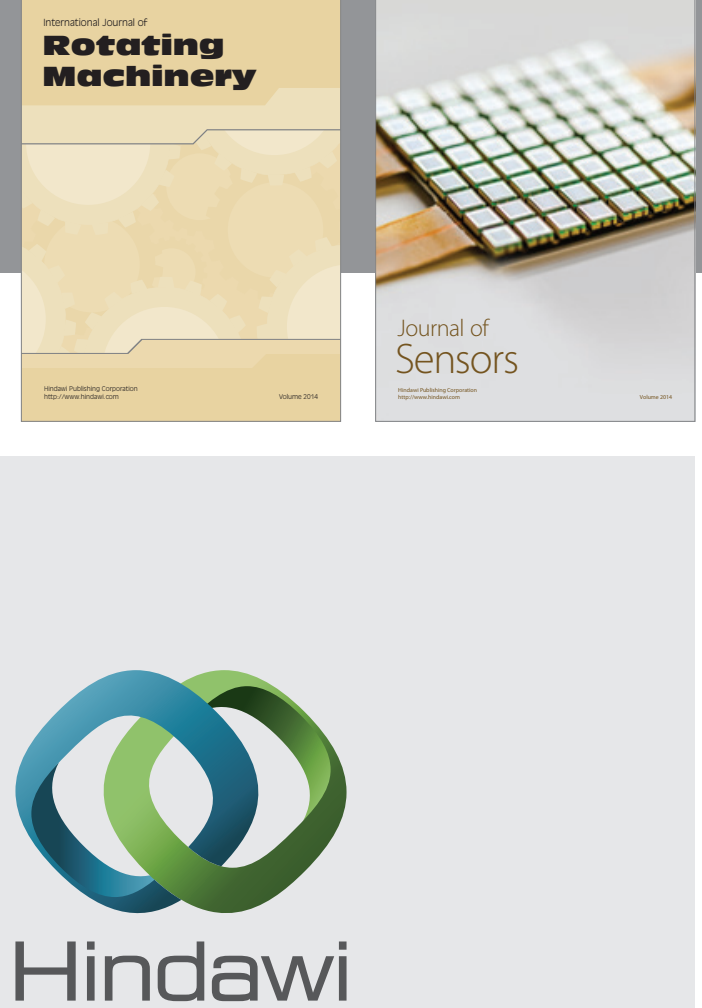

Submit your manuscripts at http://www.hindawi.com
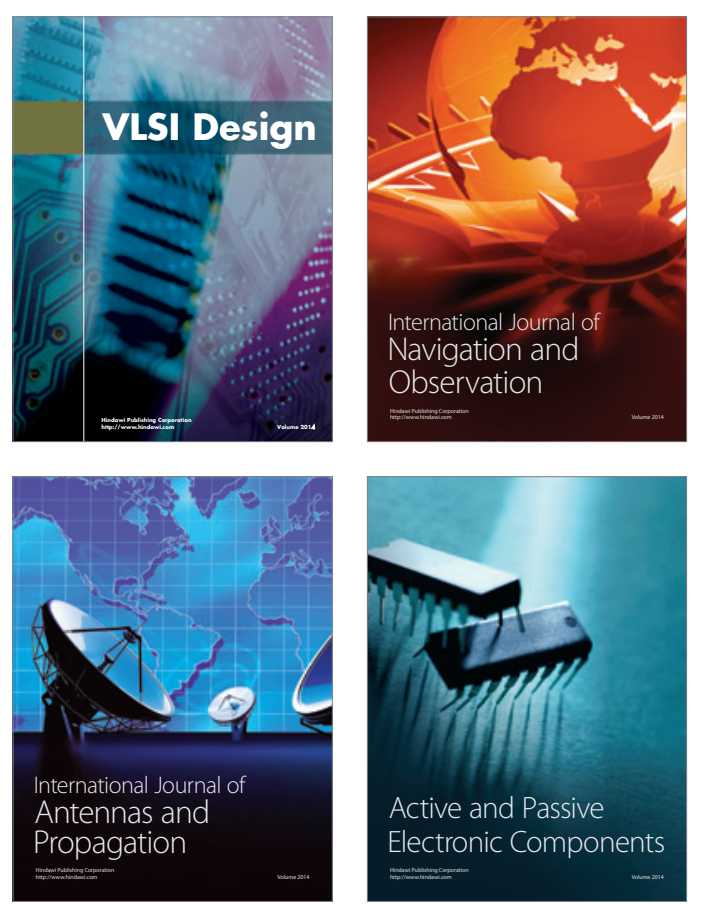
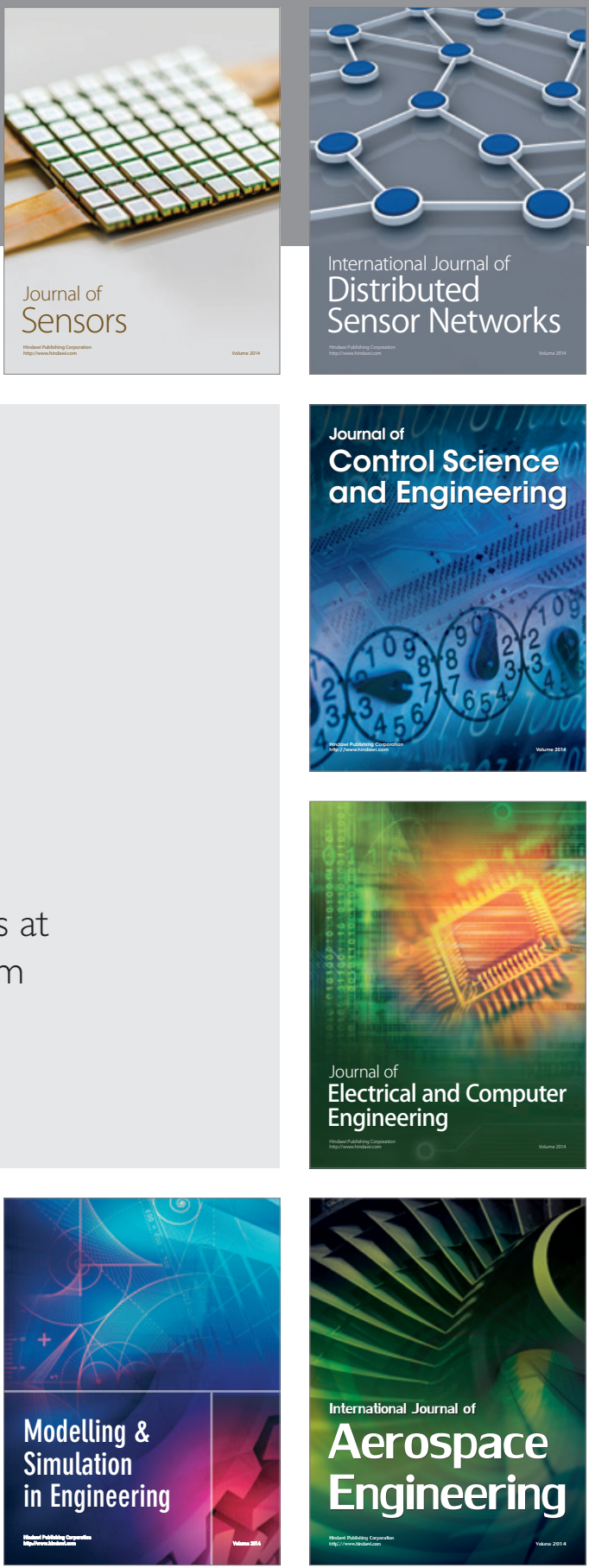

Journal of

Control Science

and Engineering
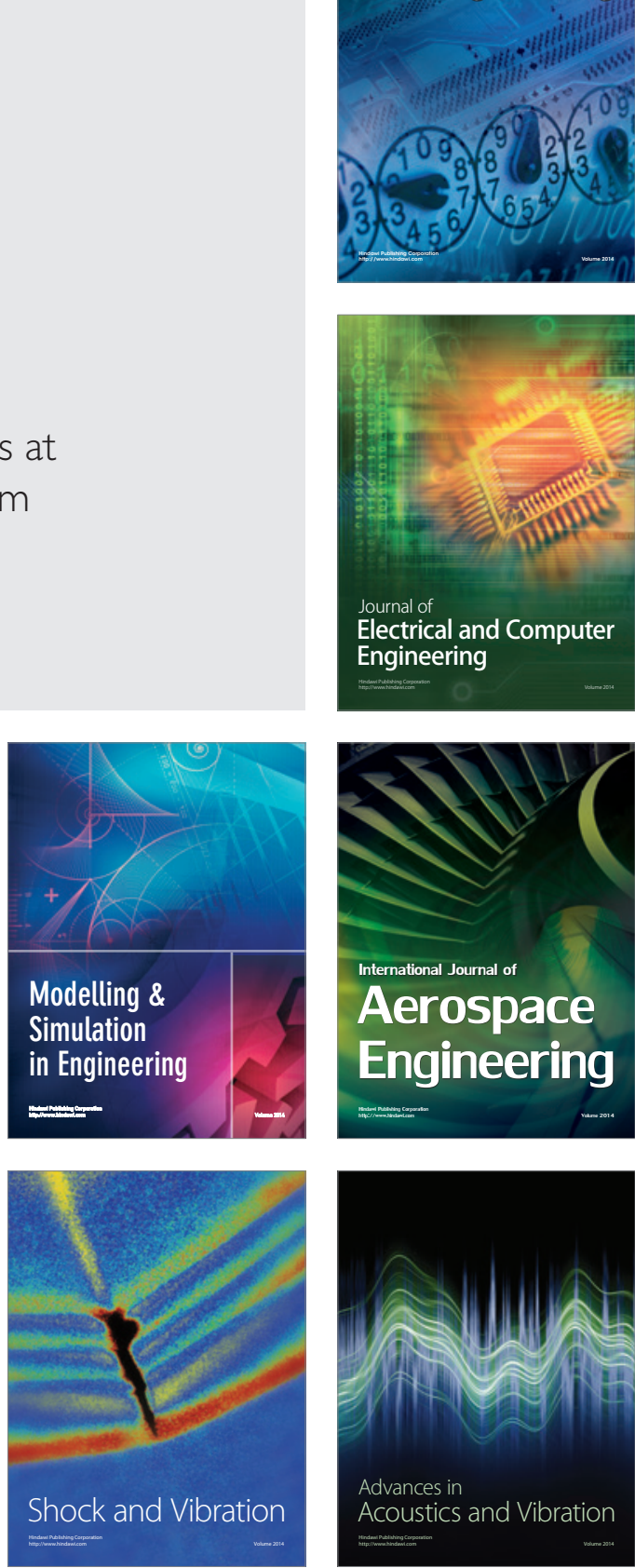